\title{
Microcatheter to Recanalization (Procedure Time) Predicts Outcomes in Endovascular Treatment in Patients with Acute Ischemic Stroke: When Do We Stop?
}

\author{
A.E. Hassan, S.A. Chaudhry, J.T. Miley, R. Khatri, S.A. Hassan, M.F.K. Suri, and A.I. Qureshi
}

\begin{abstract}
BACKGROUND AND PURPOSE: Endovascular treatment for acute ischemic stroke consists of various mechanical and pharmacologic modalities used for recanalization of arterial occlusions. We performed this study to determine the relationship among procedure time, recanalization, and clinical outcomes in patients with acute ischemic stroke undergoing endovascular treatment.
\end{abstract}

MATERIALS AND METHODS: We analyzed data from consecutive patients with acute ischemic stroke who underwent endovascular treatment during a 6-year period. Demographic characteristics, NIHSS score before and 24 hours after the procedure, and discharge mRS score were ascertained. Procedure time was defined by the time interval between microcatheter placement and recanalization or completion of the procedure. We estimated the procedure time after which favorable clinical outcome was unlikely, even after adjustment for age, time from symptom onset, and admission NIHSS scores.

RESULTS: We analyzed 209 patients undergoing endovascular treatment (mean age, $65 \pm 16$ years; 109 [52\%] men; mean admission/ preprocedural NIHSS score, $15.3 \pm 6.8)$. Complete or partial recanalization was observed in $176(84.2 \%)$ patients, while unfavorable outcome (mRS 3-6) was observed in 138 (66\%) patients at discharge. In univariate analysis, patients with procedure time $\leq 30$ minutes had lower rates of unfavorable outcome at discharge compared with patients with procedure time $\geq 30$ minutes (52.3\% versus $72.2 \%, P=.0049)$. In our analysis, the rates of favorable outcomes in endovascularly treated patients after 60 minutes were lower than rates observed with placebo treatment in the Prourokinase for Acute Ischemic Stroke Trial. In logistic regression analysis, unfavorable outcome was positively associated with age $(P=.0012)$, admission NIHSS strata $(P=.0017)$, and longer procedure times $(P=.0379)$.

CONCLUSIONS: Procedure time in patients with acute ischemic stroke appears to be a critical determinant of outcomes following endovascular treatment. This highlights the need for procedure time guidelines for patients being considered for endovascular treatment in acute ischemic stroke.

ABBREVIATIONS: $\mathrm{Cl}=$ confidence interval; $\mathrm{ICH}=$ intracerebral hemorrhage; $\mathrm{mRS}=$ modified Rankin Scale; $\mathrm{OR}=$ odds ratio; $\mathrm{PROACT}=$ Prourokinase for Acute Ischemic Stroke Trial

$\mathbf{T}$ he Brain Attack Coalition guidelines require a stroke team member at the patient's bedside within 15 minutes, initial head CT scan performed and interpreted in $<45$ minutes, and IV rtPA given within 60 minutes of arrival. ${ }^{1,2}$ Although endovascular

Received January 8, 2012; accepted after revision April 3.

From the Zeenat Qureshi Stroke Research Center, University of Minnesota, Minneapolis, Minnesota.

All authors have read and approved submission of the manuscript.

This study was performed independent of any financial support. None of the authors have any conflict of interest or financial conflicts to disclose.

Dr Qureshi has received funding from National Institutes of Health RO-1-NS44976$01 A 2$ (medication provided by ESP Pharma), the American Heart Association Established Investigator Award 0840053N, and the Minnesota Medical Foundation, Minneapolis, Minnesota.

Please address correspondence to Ameer E. Hassan, DO, Department of Neurology, Zeenat Qureshi Stroke Research Center, University of Minnesota, 12-100 PWB 516 Delaware St SE, Minneapolis, MN 55455; e-mail: ameerehassan@gmail.com treatment is a widely used option for recanalization in patients not eligible for IV rtPA, there are limited data and no current performance guidelines for endovascular treatment of acute ischemic stroke. The only recommendation states that personnel responsible for conventional angiography must arrive at the institution within 60 minutes of being notified. ${ }^{1-3}$

Recent studies have introduced the idea of "futile recanalization" after endovascular treatment of acute ischemic stroke, defined as recanalization with no improvement in outcome. ${ }^{4}$ Since then, greater emphasis has been placed on reducing the time delays in endovascular treatment to reduce the rate of futile recanalization with particular emphasis on "time to microcatheter." A re-

\footnotetext{
- Indicates open access to non-subscribers at www.ajnr.org

三 Indicates article with supplemental on-line table.

http://dx.doi.org/10.3174/ajnr.A3202
} 
cently published study identified a wide variability in time to microcatheter and delay among different institutions and concluded that there needs to be time recommendations for comprehensive stroke centers to improve acute stroke outcomes. ${ }^{5}$ However, the study was unable to demonstrate a relationship between the time interval between symptom onset and microcatheter placement and clinical outcomes. After a microcatheter is placed in a thrombus, the recanalization time varies considerably, but the clinical significance of such variations remains unclear. However, the current and proposed guidelines do not recognize the significance of the procedure time incurred after "time to microcatheter." The goal of our study was to determine the relationship of the time interval between microcatheter placement and recanalization (procedure time) and clinical outcomes among patients with acute ischemic stroke undergoing endovascular treatment.

\section{MATERIALS AND METHODS}

A retrospective study of consecutive patients with acute ischemic stroke who underwent endovascular treatment was performed between January 1, 2007 to June 1, 2010 at the University of Minnesota and Hennepin County Medical Centers and from May 2005 to July 2007 at the University of Medicine and Dentistry of New Jersey. The 3 institutions maintained a prospective endovascular procedure data base, which records information regarding the procedural components, devices used, and intraprocedural medication with doses. The protocol for collecting data was reviewed and approved by the institutional review board at each institution as part of a standardized data base.

\section{Data Collected}

We recorded the presence of cardiovascular risk factors (active smoking history, hypertension, atrial fibrillation, coronary artery disease, hyperlipidemia, diabetes mellitus, prior TIA or ischemic stroke, time interval between symptom onset and endovascular intervention, and use of IV rtPA). "Procedure time" was defined by the interval between the time of positioning the microcatheter proximal to the thrombus and the time to partial or complete recanalization (based on modified Thrombolysis in Myocardial Infarction 2 or 3 ) or procedure termination. The times were identified by 2 authors (A.E.H. and J.T.M.) individually reviewing the angiographic images and recording the times of microcatheter placement and recanalization or completion of the procedure. We also recorded admission, 24-hour posttreatment, and discharge NIHSS scores. Outcome at the time of discharge was assessed by using the mRS, ascertained from hospital discharge summaries by the vascular neurology team and occupational, speech, and physical therapists. The principal safety end points were ICH and inhospital mortality. Symptomatic ICH was defined as noncontrast CT-documented ICH resulting in neurologic deterioration $(\geq 4$ point worsening on an NIHSS score compared with previous clinical assessment). Favorable functional outcome at discharge was defined by an mRS score of $0-2$ at discharge.

\section{Technical Description for Endovascular Treatment}

In a typical procedure, patients are brought to the neuroangiography room, and through a $6 \mathrm{~F}$ introducer sheath in the common femoral artery, a $6 \mathrm{~F}$ multipurpose device-guide catheter is ad- vanced into the internal carotid artery or vertebral artery. Once the arterial occlusion is identified, a microcatheter ranging in diameter from $1.4 \mathrm{~F}$ to $2.3 \mathrm{~F}$ is advanced over a 0.014 -inch microwire into the vessel of interest, in close proximity to the thrombus. At this given moment, arterial recanalization therapy is initiated. The techniques for endovascular treatment have been described in detail in previous publications. ${ }^{6-10}$ Briefly, a combination of pharmacologic agents and/or mechanical thrombus disruption and/or retrieval is used in varying paradigms.

\section{Patient Selection}

The patient-selection criteria for endovascular treatment are described in detail in previous publications. ${ }^{7,11,12}$ Briefly, at the University of Medicine and Dentistry of New Jersey, all patients were selected for endovascular treatment on the basis of the time interval between symptom onset and emergency department presentation and the findings of noncontrast cranial CT scan. Patients with ICH or cerebral edema, sulcal effacement, and/or focal parenchymal hypoattenuation exceeding one-third of the MCA vascular territory (in those presenting after 3 hours) were excluded. At the University of Minnesota and Hennepin County Medical Centers, the patients were selected on the basis of findings of CTA and CTP scans, in addition to the noncontrast CT. The CTP protocol follows the noncontrast cranial CT scan and is discontinued if $\mathrm{ICH}$ is identified. Maps obtained from data acquired by the Brilliance 64 (Philips Healthcare, Best, the Netherlands) were generated by using Vitrea software (Vital Images, Minnetonka, Minnesota), yielding the following perfusion parameters: time to peak, mean transit time, relative $\mathrm{CBF}$, and relative $\mathrm{CBV}$. In the case of an MCA vascular territory infarction, the patient was excluded from endovascular treatment if the infarct burden was greater than or equal to one-third of the vascular territory on qualitative analysis of the relative CBV map acquired from the CTP study. ${ }^{12}$

\section{Statistical Analysis}

All data were presented by using mean \pm SD for continuous data and frequencies for categoric data. The frequency of baseline demographic and clinical characteristics, admission NIHSS score, time interval between symptom onset and endovascular treatment, and the interval between hospital presentation and endovascular treatment (time to microcatheter) were compared among patients with endovascularly treated acute ischemic stroke. We analyzed the effect of different time strata on discharge outcomes by using the receiver operating curve and goodness-offit model. We chose the smallest time interval that had an adequate number of patients resulting in a good precision estimate. We divided the patients into 2 strata based on endovascular procedure time of $\leq 30$ and $>30$ minutes. The choice of 30 minutes as the cutoff for procedure time was also supported by visual analysis of scatterplots depicting recanalization response to incremental doses in 2 of our previous dose-escalating studies. ${ }^{13,14}$ In both studies, at least half of the patients demonstrated partial or complete recanalization after administration of 4 doses of intra-arterial thrombolytics (each administered for 5 minutes) and 4 angiographic images obtained in the interim (estimated 10 minutes 


\begin{tabular}{|c|c|c|}
\hline Variable & $\begin{array}{c}\text { Odds Ratio } \\
\text { (95\% Cl) }\end{array}$ & $\begin{array}{l}P \text { Value } \\
\text { for Trend }\end{array}$ \\
\hline \multicolumn{3}{|l|}{ Age } \\
\hline$\leq 45$ years & Reference & .0012 \\
\hline $46-65$ years & $0.8(0.3-2.6)$ & \\
\hline$>65$ years & $3.6(1.2-11.3)$ & \\
\hline \multicolumn{3}{|l|}{ NIHSS score strata } \\
\hline $0-9$ & Reference & .0017 \\
\hline 10-19 & $3.3(1.3-8.3)$ & \\
\hline$\geq 20$ & $8.5(2.6-28.2)$ & \\
\hline \multicolumn{3}{|c|}{ Total procedure time (min) } \\
\hline$<30$ & Reference & .0379 \\
\hline $31-60$ & $1.3(0.6-3.3)$ & \\
\hline $61-90$ & $3.7(1.1-12.6)$ & \\
\hline$<90$ & $5.4(1.4-20.9)$ & \\
\hline
\end{tabular}

${ }^{a}$ Model adjusts for time interval between symptom onset and microcatheter placement, and recanalization status.

between each image). Therefore, there is reason to believe that 30 minutes is the earliest time to assess the procedural success.

We performed univariate analysis followed by a multivariate logistic regression model to determine the effect of procedure time on clinical outcomes at discharge. Statistical association was assessed with a $t$ test or median 2-sample test according to normality for continuous data and the $\chi^{2}$ test for categoric data. The rates of partial and complete angiographic recanalization, symptomatic and asymptomatic ICH, early neurologic improvement, favorable discharge outcome, and in-hospital mortality were compared between strata defined by procedure time. We performed multivariate logistic regression analysis, adjusting for admission NIHSS score, age, recanalization status, and the time interval between symptom onset and microcatheter placement, to determine the independent effect of procedure time strata and clinical outcomes. We estimated the procedure time after which favorable clinical outcome was unlikely (no greater than expected by the natural history of untreated ischemic stroke). Subsequently in a separate analysis, we compared rates of favorable outcome by using various cutoff values of total procedure time by using favorable rates observed in the placebo group in PROACT II as a reference as used in previous exploratory analysis (Mechanical Embolus Removal in Cerebral Ischemia trial). ${ }^{15}$ Our goal was to identify a procedure time cutoff after which the rate of favorable outcomes is not greater than what could be expected with a placebo infusion via a microcatheter placed within the thrombus. A $P$ value $<.05$ was considered significant. All analyses were performed by using SAS statistical software (SAS, Cary, North Carolina).

\section{RESULTS}

We analyzed 209 patients who underwent endovascular treatment (mean age, $65 \pm 16$ years; 109 [52\%] men; mean admission NIHSS score, $15.3 \pm 6.8$; and mean time to recanalization, $50 \pm$ 33.5 minutes). Complete or partial recanalization was observed in $176(84.2 \%)$ patients, while unfavorable outcome was observed in $138(66 \%)$ patients at discharge. Mean time from symptom onset to microcatheter was $299 \pm 110.9$ minutes.

Patients with favorable outcome had a procedure time and average time from symptom onset to recanalization that was shorter compared with patients with unfavorable outcome (41.8 minutes versus 54.3 minutes, $P=.0067$ and 330.4 minutes versus 358.8 minutes, $P=.3179$, respectively). Patients who had a procedure within 30 minutes were less likely to be women compared with those patients with a time of $>30$ minutes $(36.9 \%$ versus $52.7 \%, P=.03)$. In univariate analysis, there were no statistical differences for patients with a procedure time of $\leq 30$ minutes and those with a time of $>30$ minutes in regard to demographics, time interval between symptom onset and start of procedure, location of arterial occlusion, and admission NIHSS score (Table 1). There was no statistical difference in the rate of symptomatic and

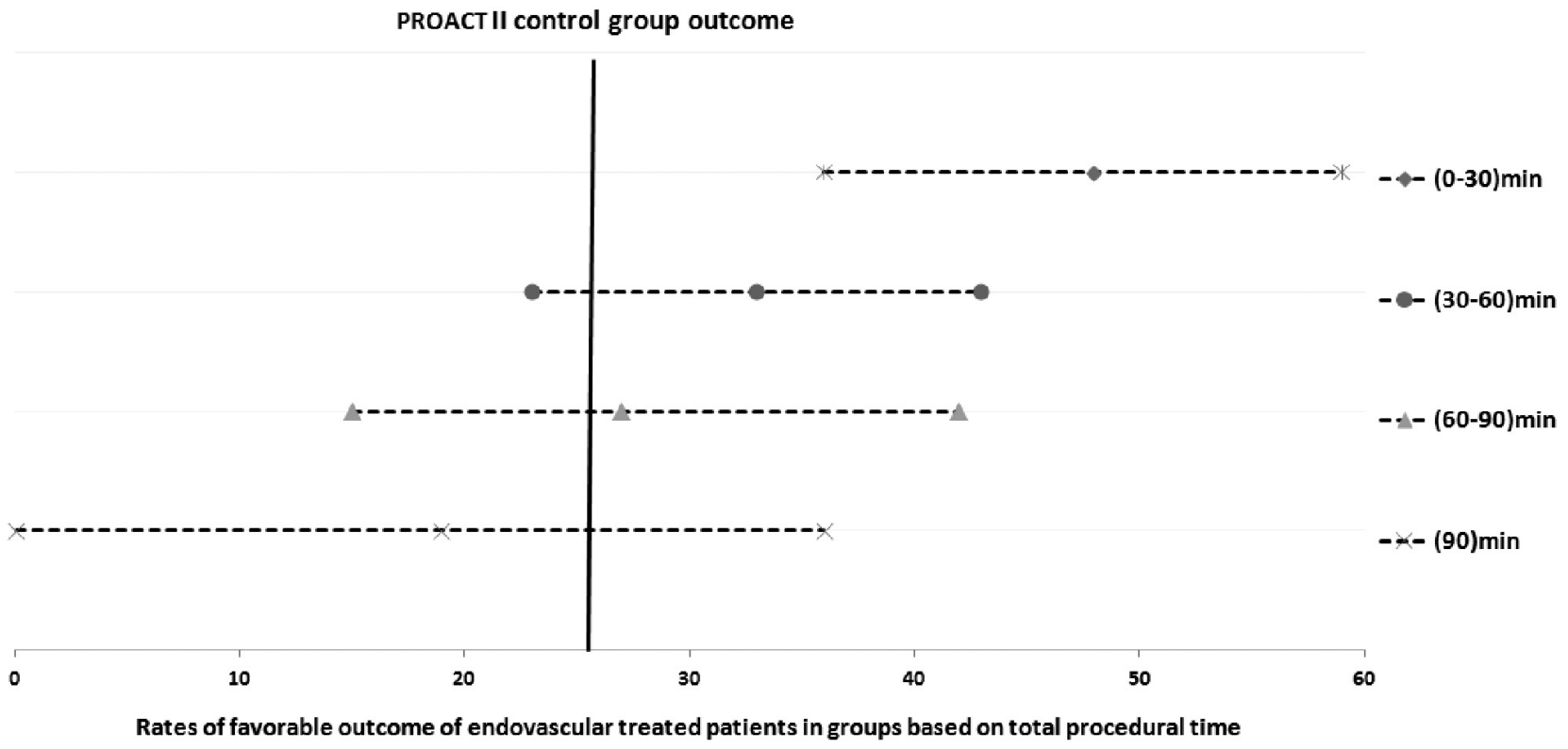

FIG 1. Rates of favorable outcome of endovascularly treated patients in groups based on total procedure time in comparison with rates observed in placebo-treated PROACT II patients. 


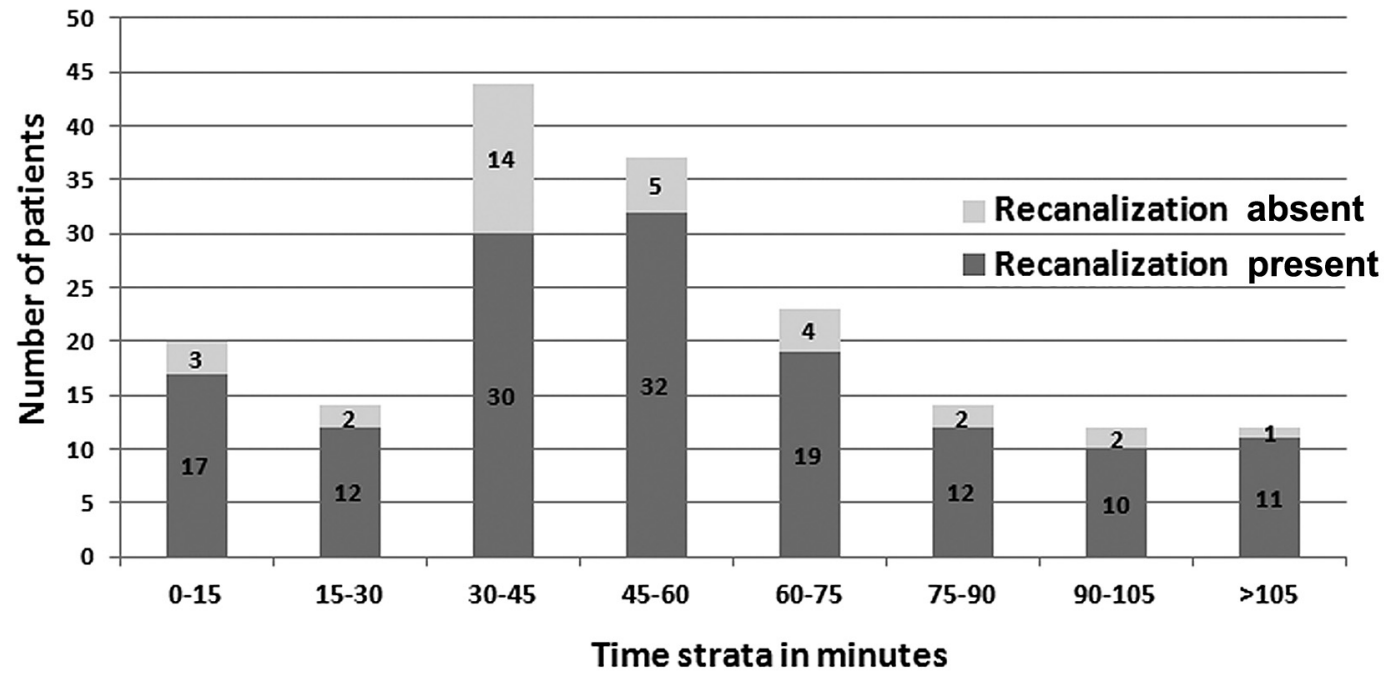

FIG 2. Rates of angiographic recanalization in endovascularly treated patients in groups based on total procedure time.

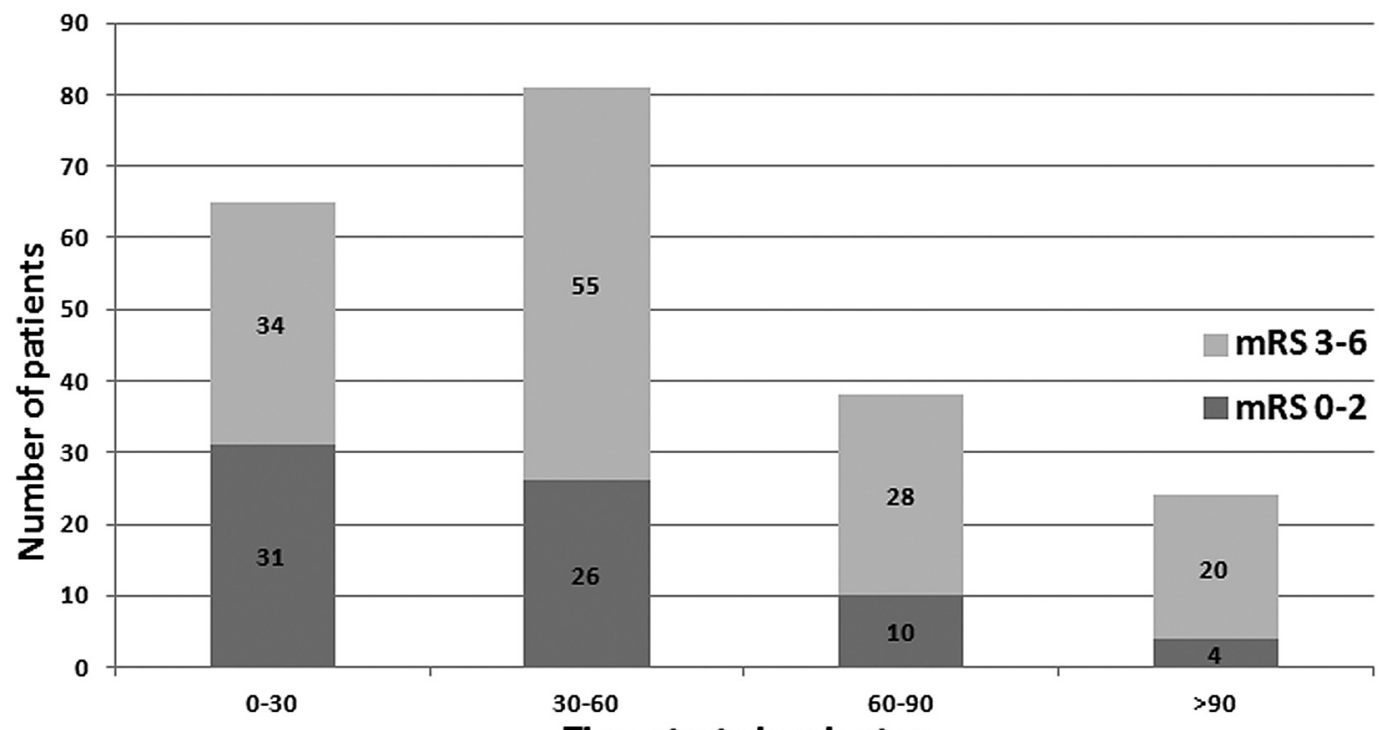

FIG 3. The relationship between the endovascular procedure time and clinical outcome at discharge.

asymptomatic ICHs between the 2 groups. Patients with procedure times of $\leq 30$ minutes had a lower rate of unfavorable outcome at discharge, defined by an $\mathrm{mRS} \geq 3$ compared with patients with a procedure time of $\geq 30$ minutes $(52.3 \%$ versus $72.2 \%, P=$ .0049).

After we adjusted for age, admission NIHSS score, recanalization status, and time interval between the onset of symptoms and microcatheter placement, the longer procedure times of 61-90 minutes (OR, 3.7; 95\% CI, 1.1-12.6) and >90 minutes (OR, 5.4; 95\% CI, 1.4-20.9) were associated with higher rates of unfavorable outcome compared with microcatheter-to-recanalization time of $\leq 30$ minutes with a $P$ value for trend $(P=.0379)$ (Table). Increased age $(P=.0012)$ and NIHSS score $(P=.0017)$ at admission were independent predictors of unfavorable outcome.

In our analysis, favorable outcomes in endovascularly treated patients after 60 minutes were comparable with those in the PROACT-II control group (Fig 1). To explore the relationship between clinical outcome recanalization, and time, we plotted the rate of angiographic recanalization and time strata (Fig 2), and plotted the unfavorable outcomes and time strata (Fig 3). The rate of unfavorable outcome was much higher in the 60-90 and $>90$ minute strata.

\section{DISCUSSION}

This study highlights the relationship between microcatheter placement and recanalization (procedure time) and clinical outcomes among patients with acute ischemic stroke undergoing endovascular treatment. To our knowledge, procedure time (as defined by time from microcatheter to recanalization/procedure completion) had not been previously studied. Procedure time in patients undergoing endovascular treatment demonstrates considerable variation within procedures, and such variations directly impact clinical outcome.

There are multiple factors that determine the outcome of endovascular treatment in patients with acute ischemic stroke. Pre- 
vious studies have focused heavily on achieving recanalization and using recanalization as a measure of treatment success. ${ }^{4}$ However, the recognition of futile recanalization has shifted the focus of treatment to time-efficient protocol implementation. In a recently published multicenter study of consecutive patients with acute ischemic stroke who underwent endovascular treatment, the authors compared the clinical outcomes based on recanalization. ${ }^{4}$ A large proportion (49\%) of patients who had successful recanalization did not achieve favorable outcomes, presumably due to irreversible ischemic injury not detected by noncontrast CT scan. Presumably, early recanalization by reducing the delays in treatment could reduce the rate of futile recanalization. The Interventional Management of Stroke investigators found that time to angiographic reperfusion and age predicted good clinical outcome in those patients who had angiographic reperfusion. ${ }^{16}$ The probability of good clinical outcome decreased as the time to reperfusion increased and then approached that of patients without angiographic reperfusion. After analyzing our data and adjusting for age, NIHSS score, and time interval between onset and microcatheter placement, we found that procedure time was still a predictor of unfavorable outcome in patients who had recanalization.

In the acute ischemic stroke setting, centers follow the Brain Attack Coalition guidelines for time-efficient administration of IV rtPA. Stroke centers continuously monitor door to CT scan and door to needle as quality parameters and implement strategies to improve performance. In theory, the same principles should apply to endovascular treatment. However, the basic elements necessary for recognition and improvement have not been identified either through the consensus of experts or clinical studies. The comprehensive stroke center metrics ${ }^{17}$ identify "time to microcatheter" as a recommended metric to monitor but did not specify the thresholds for acceptable performance. The selection of time to microcatheter was based on a similar performance metric, "door to balloon," in patients with acute myocardial infarction. The American College of Cardiology and the American Heart Association guidelines for the management of ST-elevation myocardial infarction state that percutaneous coronary intervention should be performed as quickly as possible, preferably within 90 minutes from the moment of first medical contact. ${ }^{18}$ Although there are similarities between stroke and myocardial infarction treatment, procedure times in stroke have much larger variations due to considerable variability in tortuosity, vessel location, and the nature of the occlusion. ${ }^{14,16}$ Such variability in procedure time in patients with acute stroke, unlike those with myocardial infarction, increases the likelihood of a prominent relationship to outcomes.

The effect of shorter procedure time on patient clinical outcome is provocative, but it needs to be interpreted with caution. Although we found that 42 minutes was our mean procedure time in patients with favorable outcomes, the evidence does not support aborting procedures after that timeframe. Every endovascular procedure needs to be looked at individually, incorporating patient- and treatment-related factors in the decision-making. Centers should begin monitoring procedure times, and guidelines need to be provided to standardize treatment protocols if procedure time exceeds 60 minutes. Endovascular treatments that might restore blood flow more rapidly, such as mechanical intervention, flow-diversion devices, or the combination of thrombolytics, should be incorporated into treatment paradigms. However, aggressive manipulation may lead to unfortunate complications including dissection, perforation, ${ }^{19}$ vessel rupture, ${ }^{20}$ and conversion of distal emboli to more proximal larger artery occlusions. ${ }^{21}$ There have been discussions about procedure time as a marker of extensive occlusions, but studies have shown that clot volume in acute ischemic stroke is not related to recanalization. ${ }^{20-22}$

The retrospective nonrandomized nature of the study is a limitation. IV rtPA was not withheld in any patients, consistent with current guidelines. There are limitations to the use of the NIHSS score and $\mathrm{mRS}$ in describing patient deficits and disabilities. The NIHSS score, in particular, provides limited information in patients with deficits referable to the posterior circulation. The bias introduced may be more pronounced in our and other studies that include posterior circulation ischemic events unlike studies such as PROACT I and II, which only included patients with anterior circulation ischemic events. ${ }^{15}$ There are also differences in time points of mRS ascertainment. Comparison of outcomes between our study and placebo-treated patients in the PROACT trial may be further limited by improvements in poststroke medical management since PROACT II was completed. Therefore, the comparison between the findings of our study and PROACT II is only exploratory. Prestroke mRS or NIHSS scores were not documented consistently, potentially undermining the benefit of treatment among patients with prestroke neurologic deficits by inclusion of patients with pre-existing disability and poor mRS scores. ${ }^{3-5}$ We used mRS and NIHSS scores that were documented for 7 days or at discharge but did not have 3-month functional outcomes or mortality rates. Broderick et $\mathrm{al}^{23}$ found that a given level or amount of change in the NIHSS score during the first 24 hours and the NIHSS score at 7-10 days following treatment was the most sensitive measure for detecting differences in the effectiveness of thrombolytic treatment during the first 3 months after an ischemic stroke. The prognostic value of 24-hour and 7-day data in that analysis validates the outcomes used in our study.

\section{CONCLUSIONS}

Our observations highlight the need for specific time guidelines to reduce the delays in endovascular treatment among patients with acute ischemic stroke. Procedure time in patients undergoing endovascular treatment for acute ischemic stroke appears to be a critical determinant of outcomes following treatment. Although we recommend procedure times of $<60$ minutes, every endovascular procedure needs to be looked at individually, incorporating patient- and treatment-related factors in the decision-making. Comprehensive stroke centers should begin monitoring procedure times, and special considerations may be required if procedure time exceeds 60 minutes. Further studies need to standardize the definition of procedure time and determine the effect of various thresholds to improve outcomes of patients with acute ischemic stroke. 


\section{REFERENCES}

1. Alberts MJ, Hademenos G, Latchaw RE, et al. Recommendations for the establishment of primary stroke centers: Brain Attack Coalition. JAMA 2000;283:3102-09

2. Alberts MJ, Latchaw RE, Selman WR, et al. Recommendations for comprehensive stroke centers: a consensus statement from the Brain Attack Coalition. Stroke 2005;36:1597-616

3. Schriger DL, Kalafut M, Starkman S, et al. Cranial computed tomography interpretation in acute stroke: physician accuracy in determining eligibility for thrombolytic therapy. JAMA 1998;279: 1293-97

4. Hussein HM, Georgiadis AL, Vazquez G, et al. Occurrence and predictors of futile recanalization following endovascular treatment among patients with acute ischemic stroke: a multicenter study. AJNR Am J Neuroradiol 2010;31:454-58

5. Miley JT, Memon MZ, Hussein HM, et al. A multicenter analysis of "time to microcatheter" for endovascular therapy in acute ischemic stroke. J Neuroimaging 2011;21:159-64

6. Burns TC, Rodriguez GJ, Patel S, et al. Endovascular interventions following intravenous thrombolysis may improve survival and recovery in patients with acute ischemic stroke: a case-control study. AJNR Am J Neuroradiol 2008;29:1918-24

7. Qureshi AI, Janjua N, Kirmani JF, et al. Mechanical disruption of thrombus following intravenous tissue plasminogen activator for ischemic stroke. J Neuroimaging 2007;17:124-30

8. Qureshi AI, Harris-Lane P, Kirmani JF, et al. Intra-arterial reteplase and intravenous abciximab in patients with acute ischemic stroke: an open-label, dose-ranging, phase I study. Neurosurgery 2006;59: 789-96, discussion 96-97

9. Qureshi AI, Siddiqui AM, Kim SH, et al. Reocclusion of recanalized arteries during intra-arterial thrombolysis for acute ischemic stroke. AJNR Am J Neuroradiol 2004;25:322-28

10. Qureshi AI, Hussein HM, Abdelmoula M, et al. Subacute recanalization and reocclusion in patients with acute ischemic stroke following endovascular treatment. Neurocrit Care 2009;10:195-203

11. Qureshi AI, Suri MF, Ali Z, et al. Intraarterial reteplase and intravenous abciximab for treatment of acute ischemic stroke: a preliminary feasibility and safety study in a non-human primate model. Neuroradiology 2005;47:845-54.
12. Hassan AE, Zacharatos H, Rodriguez GJ, et al. A comparison of computed tomography perfusion-guided and time-guided endovascular treatments for patients with acute ischemic stroke. Stroke 2010; 41:1673-78

13. Qureshi AI, Suri MF, Shatla AA, et al. Intraarterial recombinant tissue plasminogen activator for ischemic stroke: an accelerating dosing regimen. Neurosurgery 2000;47:473-76, discussion 477-79

14. Qureshi AI, Ali Z, Suri MF, et al. Intra-arterial third-generation recombinant tissue plasminogen activator (reteplase) for acute ischemic stroke. Neurosurgery 2001;49:41-48, discussion 48-50

15. Furlan A, Higashida R, Wechsler L, et al. Intra-arterial prourokinase for acute ischemic stroke: the PROACT II study-a randomized controlled trial. Prolyse in Acute Cerebral Thromboembolism. JAMA 1999;282:2003-11

16. Khatri P, Abruzzo T, Yeatts SD, et al. Good clinical outcome after ischemic stroke with successful revascularization is time-dependent. Neurology 2009;73:1066-72

17. Leifer D, Bravata DM, Connors JJ 3rd, et al. Metrics for measuring quality of care in comprehensive stroke centers: detailed follow-up to Brain Attack Coalition comprehensive stroke center recommendations-a statement for healthcare professionals from the American Heart Association/American Stroke Association. Stroke 2011; 42:849-77

18. Derex L, Adeleine P, Nighoghossian N, et al. Factors influencing early admission in a French stroke unit. Stroke 2002;33:153-59

19. Nguyen TN, Lanthier S, Roy D. Iatrogenic arterial perforation during acute stroke interventions. AJNR Am J Neuroradiol 2008;29: 974-75

20. Shi ZS, Liebeskind DS, Loh Y, et al. Predictors of subarachnoid hemorrhage in acute ischemic stroke with endovascular therapy. Stroke 2010;41:2775-81

21. Nguyen TN, Babikian VL, Romero R, et al. Intra-arterial treatment methods in acute stroke therapy. Front Neurol 2011;2:9

22. Qureshi AI, Alkawi A, Hussein HM, et al. Angiographic analysis of intravascular thrombus volume in patients with acute ischemic stroke. J Endovasc Ther 2007;14:475-82

23. Broderick JP, Lu M, Kothari R, et al. Finding the most powerful measures of the effectiveness of tissue plasminogen activator in the NINDS tPA stroke trial. Stroke 2000;31:2335-41 